\title{
CONCEPTO Y NACIONALIDAD DEL LAUDO
}

\author{
Felipe Cuberos $\left(^{*}\right)$
}

\section{INTRODUCCIÓN}

Dada la extraordinaria precariedad existente en los distintos ordenamientos en cuanto a la noción de laudo y a su caracterización como nacional o extranjero, resulta al menos pertinente preguntarse si, de algún modo, existe algún tipo de consenso internacional en torno a estos conceptos, y sin son ellos en alguna medida relevantes para el cabal entendimiento de la institución arbitral en el mundo de hoy. En efecto, viendo que la justicia arbitral es cada vez más cercana a la vida de los negocios y que por tanto, el elemento "laudo" está cada vez más presente en la definición de relaciones jurídicas, y considerando además que estamos en un entorno globalizado donde es cada día más frecuente que haya pronunciamientos emitidos en un lugar geográfico o bajo una determinada legislación pero que, a su turno, estén llamados a ejecutarse en otras latitudes, parece prudente definir, o por lo menos, tratar de entender qué es lo que hace que un "laudo" sea laudo, y qué elementos, desde la perspectiva de la teoría jurídica, permiten establecer su nacionalidad para, a partir de ello, derivar los efectos que en derecho correspondan.

Dentro del contexto antes expuesto, el presente escrito pretende entonces hacer algunas reflexiones en torno al concepto y nacionalidad del laudo, haciendo pues la salvedad de que, no habiendo en la mayoría de las legislaciones e instrumentos internacionales una definición técnica ni mucho menos uniforme de lo que es un laudo, ni habiendo reglas claras en torno a su nacionalidad, existe un amplio margen de interpretación para dilucidar su significado, características y alcances. Así las cosas, y 
desde luego, sin pretender ser exhaustivos en ello, nuestra intencion es formular planteamientos que contribuyan a un mejor entendimiento de estas nociones y de sus implicaciones para la efectividad, en una determinada jurisdicción, de las decisiones que los árbitros hubieren podido adoptar por fuera de su ámbito geográfico y procesal.

\section{Concepto de Laudo}

Resulta curioso, por decir lo menos, que siendo de común aceptación que el pronunciamiento que pone fin a una disputa arbitral se denomina "laudo" ${ }^{1}$ los instrumentos internacionales que existen sobre la materia no se hayan ocupado en regular de manera detallada su definición, características y alcances. En efecto, una simple observación desprovista de mayores análisis Ileva fácilmente a concluir que los principales tratados y reglamentos internacionales en materia de arbitraje, antes que detenerse en la regulación del "laudo", se han ocupado más bien en dibujar cuál es el camino para someterse al juicio de árbitros; cuál el procedimiento que deben ellos seguir para conocer del asunto, y cómo, finalmente, logra hacerse efectiva la decisión de los árbitros.

La situación antes descrita, es decir, la ausencia de una tipificación clara de qué es un "laudo", no es fruto de la casualidad sino que, por el contrario, puede encontrar su sustento en el origen mismo de la institución arbitral, tanto en su concepción histórica como en su base jurídica. Ciertamente, la noción de arbitraje parte del más elemental de los principios, cual es el que las partes de un conflicto, al verse imposibilitadas para resolver por

1. Eso, desde luego, sin entrar por ahora en la discusión acerca de si esa denominación también le cabe a otros pronunciamientos que puedan emitirse dentro del trámite arbitral que, al menos en Colombia, parece ya no tener cabida dados los reiterados pronunciamientos jurisprudenciales en sentido contrario. Al efecto, véanse por ejemplo la Sentencia de Unificación SU-174 de 2007 de la Corte Constitucional o el fallo de exequátur proferido mediante Sentencia del 30 de enero de 2004 de la Sała de Casación Civil de la Corte Suprema de Justicia. 
sí solas la controversia, no tienen otro camino que delegar en un tercero, normalmente escogido por ellas mismas, la definición de una solución en forma equitativa, ágil y segura. Este principio, reconocido desde las civilizaciones más antiguas, ha tenido desde luego una evolución que lo ha venido haciendo cada vez más sofisticado, y si se quiere, también más complejo. Así por ejemplo, en las sociedades primitivas, los miembros más importantes de la comunidad, tras haber intentado propiciar un arreglo pacífico entre las partes, terminaban por regular la "composición" que uno de los involucrados en el conflicto debía pagar al otro2; igualmente, en el derecho romano, había una etapa denominada "in judicio" para la solución de conflictos, que se adelantaba ante un juez privado designado por las partes; en la edad media, fue muy común la realización de juicios ante los líderes de agremiaciones profesionales, y ya en la edad moderna, en diversas ordenanzas francesas y españolas, se hacen repetidas alusiones a la solución de conflictos por terceros escogidos por las partes a fin de "evitar pleitos largos y costosos capaces de arruinar a todos", tal como se menciona en las Ordenanzas de Bilbao de $1737^{3}$.

Así las cosas, y sin entrar pues aquí en juicios de valor acerca de las bondades de la justicia arbitral, puesto que no es ese el objeto de nuestro estudio, lo que sí es claro es que la institución arbitral supone una "sustitución" voluntaria de las partes por uno o varios terceros que las reemplazan para la solución del conflicto, ante lo cual resulta lógico pensar que si las partes mismas hubieran podido alcanzar cualquier tipo de solución sin mayores trabas ni formalismos, también así deberían poder hacerlo sus delegados. Bajo esta perspectiva, se entiende entonces cómo la escasa reglamentación que existe para la decisión arbitral propiamente dicha es fiel al origen eminentemente voluntario y contractual de la institución, ante lo cual es claro que es más bien la excesiva reglamentación actual del proceso (y

2. Monroy Cabra, Marco Gerardo - "Arbitraje Comercial", Editorial Temis, Bogotá, 1982.

3. Ver a este respecto el capítulo introductorio de la obra "Panorama y Perspectivas de la Legislación Iberoamericana sobre Arbitraje Comercial" - Cámara de Comercio de Bogotá, 1993. 
no la ausencia de definición en cuanto a qué es un laudo) la que va en contra de la naturaleza misma del procedimiento arbitral.

Ante la ausencia de una tipificación internacional clara y uniforme alrededor de la noción de "laudo", la tendencia generalizada ha sido la de darle a éste un significado y alcance que, o bien pueden ser acordes con su simple acepción idiomática, o bien pueden guardar correspondencia con lo que al efecto prevén las distintas legislaciones nacionales que, en muchos casos, son igualmente ambiguas en torno a la definición. A continuación entonces presentamos una visión general de estas dos concepciones de "laudo" (es decir, la linguiística y la normativa basada en las legislaciones nacionales), para luego hacer algunas reflexiones en cuanto a su posible aplicación en el contexto internacional.

\subsection{Noción Idiomática y legislaciones nacionales}

Desde un punto de vista puramente lingüístico, parecería no haber duda en cuanto a que el laudo es, en efecto, la decisión final de la controversia sometida al procedimiento arbitral. Así lo confirma el Diccionario de la Real Academia Española que, al referirse a la definición de laudo, simplemente se limita a establecer que es la "...Decisión of fallo que dictan los árbitros o amigables componedores..." 4 . A pesar de lo escueta de la definición, y haciendo abstracción de su extensión a las decisiones tomadas por "amigables componedores", por cuanto ello no parecería muy preciso a la luz de las más modernas concepciones sobre métodos alternativos de solución de conflictos, to que vale la pena rescatar aquí es que se define el laudo como un "fallo" que, a su turno, es la "...Sentencia de un juez o de un tribunal, y en ella, especialmente, el pronunciamiento decisivo o imperativo..."5, ante lo cual es evidente que aparecen ya dos elementos de importancia capital para la institución objeto de nuestro análisis: el primero, que

4. Real Academia Española - Diccionario de la Lengua Española, Vigésima Segunda Edición, España, 2001 .

5. Real Academia Española, ibidem. 
se trata de la decisión de alguien investido de jurisdicción; el segundo, que se trata de la decisión final y definitiva, es decir, de aquella cuyo objeto es ya definir el fondo del asunto, poniendo con ello fin a la controversia que hubiere surgido entre las partes.

La tendencia a calificar como laudo a la "decisión definitiva" (y vinculante) de los árbitros ha estado presente de manera más o menos constante en diversas legislaciones herederas de las tradiciones jurídicas más disímiles. En efecto, aún cuando muchos ordenamientos - incluido el colombiano - carecen de lo que técnicamente podría considerarse una definición de laudo, sí incluyen elementos que son demostrativos de su tratamiento. Veamos algunos ejemplos: En primer término, puede resultar interesante ilustrar el punto con una normatividad no solo muy antigua (ya que proviene de la segunda mitad del Siglo XIX), sino que además es ajena por completo a nuestra tradición legislativa. Se trata de la legislación japonesa, seguidora cercana de la tradición alemana en esta y otras muchas materias, donde se regula que "...el laudo arbitral tendrá entre las partes el mismo efecto que un juicio final y definitivo de una corte..." 6 . De igual forma, y pasándonos a algo más cercano, aunque proveniente de una cultura legal igualmente ajena a la nuestra, en los Estados Unidos, quizás siguiendo la tendencia inglesa de tener la decisión proferida por etapas, se utiliza la expresión "preaward", reservando la expresión "award" o laudo a aquello que revista un carácter definitivo o si se quiere, definitorio ${ }^{7}$.

Ahora bien; trayendo el análisis al otro extremo, es decir, a una legislación moderna y completamente afín a nuestra formación jurídica, encontramos que la ley española de arbitraje, proferida el 23 de diciembre de 2003, si bien no incluye un principio tan categórico como el de la normativa japonesa, sí parece ser partícipe del mismo. En efecto, la reglamentación del laudo aparece a partir del Título VI, que se refiere a "la terminación de las

6. Articulo 800 del Código de Procedimiento Civil Japonés.

7. Uniform Arbitration Act. En cualquier caso, hay que tener presente que en la legislación británica de 1996, las decisiones que se adoptan en las distintas etapas del proceso sí son calificadas como laudos. 
actuaciones" dentro del procedimiento arbitral, lo cual, aunado al tenor de las disposiciones que allí se incluyen, es sin duda demostrativo de que con el laudo comienza el fin del proceso. No obstante lo anterior, no podemos desconocer tampoco que en la moderna aproximación adoptada por la denominada "Ley 60 de 2003" del país ibérico, hay también espacio para considerar como laudo algo que no sea definitivo, final y concluyente. Así por ejemplo, el artículo 37 de la ley consagra que "...los árbitros decidirán la controversia en un solo laudo o en tantos laudos parciales como estimen necesarios..." (subrayado fuera del texto), y que, en la misma línea, el artículo 38 contempla que "...los árbitros cesarán en sus funciones con el laudo definitivo..." (subrayado fuera del texto). Sea de ello lo que fuere, lo que sí parece claro es que lo único susceptible de calificarse como laudo (sea éste parcial o definitivo) a la luz de la legislación española es un pronunciamiento tendiente ya a resolver el fondo del asunto, es decir, a "decidir la controversia", según las voces del ya transcrito aparte del artículo 37 de la ley, lo cual hace eco de lo ya establecido en la legislación francesa en cuanto a que el "laudo" tiene efectos de cosa juzgada 8 , y por lo mismo, no hace sino reafirmar el principio que hemos venido comentando, es decir, aquel en virtud del cual el laudo es, en principio y como regla general', la decisión que resuelve el fondo del asunto y no otra diferente.

Pasando a otras normativas adoptadas en distintos momentos por varios países de América Latina, encontramos que el Código de Procedimiento Civil y Comercial de la Nación Argentina, al referirse al laudo, estableció que "...los árbitros pronunciarán su fallo sobre todas las pretensiones sometidas a su decisión..." 10 ; en la legislación guatemalteca, la norma hace referencia a "Laudo Definitivo" lo cual, aunque en principio deja la impresión de que no todo laudo es definitivo, visto en su contexto permite concluir que las referencias legales al laudo aluden a

8. Artículo 1475 del Código de Procedimiento Civil Francés.

9. Desde luego, como toda regla general, tiene sus excepciones y variantes pero para efectos de este escrito nos concentramos en ésta, que es la tendencia más difundida según queda demostrado.

10. Artículo 754 del Código de Procedimiento Civil y Comercial de la Nación Argentina. 
una decisión final ${ }^{11}$; y en países como México o Costa Rica, se ha exigido que el laudo sea "fundamentado", todo lo cual, una vez más, muestra la tendencia general a aceptar que el laudo arbitral es la decisión que pone fin al proceso, así no se le haya definido como tal.

\subsection{El laudo en el ámbito internacional}

Como ya lo advertimos, en el campo internacional la tendencia ha sido la de plegarse a lo establecido en las legislaciones domésticas para la conceptualización del elemento "laudo", dada la falta de claridad de los instrumentos existentes al respecto. No obstante lo anterior, en materia internacional existen algunas particularidades que vale la pena discutir. Veamos:

En primer término, la comunidad internacional ha aceptado que los laudos, en el contexto de un arbitraje internacional, no necesariamente tienen que ser motivados ${ }^{12}$. Esto, que bajo una óptica puramente doméstica sería inaceptable (y que eventualmente podría tomarse como demostrativo de que no hay allí un pronunciamiento final y definitivo sobre el fondo del asunto) reitera un principio de relativa aceptación en el arbitraje internacional fundamentalmente por dos razones: la primera, porque el arbitraje internacional es en todos sus aspectos más flaco en formalismos que los arbitrajes nacionales; la segunda, porque en los negocios internacionales (que son los que de suyo dan lugar a arbitrajes internacionales), resulta preferible proteger el buen nombre comercial de los actores del conflicto que justificar las razones por las que una $\mathbf{u}$ otra parte pueda verse obligada a atender los reclamos de la otra. En otras palabras, los jugadores del comercio internacional, ante la posibilidad de

11. Articulo 41 del Decreto 67-95.

12. Esto, desde iuego, sin perjuicio de lo que establecen algunos reglanentos de arbitraje internacional, como el de la Cámara de Comercio Internacional ("CCl"), cuyo artículo 25 es bien claro en establecer que "... el latudo deberá ser motivado...". 
verse expuestos a un fallo cuyos fundamentos puedan demostrar con elocuencia su mal proceder en los negocios, en ocasiones prefieren simplemente permitir que los árbitros lleguen al fondo del asunto sin extenderse en mayores argumentos explicativos de su decisión. Por esta razón, si bien la motivación de los laudos no es per se extraña al arbitraje internacional (ver nota de pie de página número 12 anterior), para que ella se produzca normalmente (aunque no siempre ni bajo todos los reglamentos internacionales) debe haber una solicitud de las partes en ese sentido.

En segundo lugar, la terminología de algunos instrumentos internacionales difiere un poco de la utilizada en las legislaciones nacionales, sin que pueda a ciencia cierta establecerse si ello ocurre como parte de un esfuerzo deliberado de dichos instrumentos por brillar con luz propia para diferenciarse de los ordenamientos nacionales creando en torno suyo unos conceptos e instituciones particulares, o si por el contrario, de lo que se trata es simplemente de discrepancias meramente semánticas donde, a pesar de la diferencia en los términos utilizados, los fenómenos reglamentados son los mismos que, con otras denominaciones, aparecen también en las regulaciones domésticas de los estados. Así por ejemplo, la Convención de Nueva York de 1958 (concebida para facilitar el reconocimiento y ejecución en un estado de las decisiones arbitrales proferidas en otro estado) utiliza desde su título mismo la expresión "Sentencias Arbitrales" que, además de ser una expresión de poca o ninguna utilización en las codificaciones de alcance nacional, no se encuentra definida en la misma convención que la invoca. Ante esta circunstancia, bien cabe plantearse si esas "sentencias" son el mismo laudo arbitral al que nos hemos venido refiriendo, o si, por el contrario, la expresión tiene una connotación diferente y es susceptible de extenderse a otro tipo de pronunciamientos. Partiendo una vez más del contenido lingüístico, encontramos que la palabra "Sentencia" en su tercera acepción alude a una "...declaración del juicio y resolución del juez...", y que solo acompañada de la palabra "definitiva" es reconocida en castellano como "...aquella en 
que el juzgador, concluido el juicio, resuelve finalmente sobre el asunto principal..."13 (subrayado fuera del texto).

Visto lo anterior, e independientemente de lo exótica que eventualmente pueda aparecer esta aplicación de la Convención de Nueva York, no parecería descontextualizado sostener que es este un instrumento diseñado para permitir, no solo el reconocimiento y ejecución de los "laudos" o decisiones arbitrales definitivas provenientes del exterior, sino también de cualquier otra decisión arbitral (así no sea la final y definitiva) que pueda producirse en un país diferente de aquel en el que ha de aplicarse. Es más; el numeral 2 del Artículo I de la Convención sostiene que "...la expresión "Sentencia Arbitral" no solo comprenderál las sentencias dictadas por los árbitros nombrados para casos determinados, sino también las sentencias dictadas por los órganos arbitrales permanentes a los que las partes se hayan sometido...". Si se tiene en cuenta que esos "órganos arbitrales permanentes" suelen ser más unos administradores de procedimientos que unos falladores investidos de jurisdicción para definir el fondo del asunto, es claro que, al menos en su finalidad, lo que muy seguramente quisieron los redactores de la Convención de Nueva York fue dejar establecida su aplicabilidad también para decisiones de mero trámite y no solamente para el pronunciamiento definitivo dirimente de la controversia.

Ahora bien; si en la práctica se ha visto más la aplicación de la Convención de Nueva York a "sentencias definitivas" que a otro tipo de decisiones (cosa que tenemos que admitir como cierta), ello se debe precisamente a la tendencia, que ya comentamos, de entender la terminología de los instrumentos internacionales con la misma óptica y bajo la misma perspectiva delineada por los ordenamientos nacionales donde, como ya se anotó, es aparentemente fácil concluir que el laudo arbitral es considerado en las distintas legislaciones domésticas, y con algunas variantes, como el pronunciamiento definitivo de los árbitros.

13. Real Academia Española, ibidem 
El otro instrumento importante para el reconocimiento y la ejecución en Colombia de decisiones arbitrales extranjeras es la Convención de Panamá, donde el punto que comento parece un tanto más pacífico, por cuanto hay artículos en ella que se refieren a "...las sentencias o laudos arbitrales..." 14 , y por cuanto además, allí mismo se establece que tales pronunciamientos "....tendrán fuerza de sentencia judicial ejecutoriada...". En otras palabras, aún cuando la Convención de Panamá tampoco se detiene en definir el "laudo", sí es precisa en la determinación de su alcance.

Finalmente, no está de más hacer una breve referencia al Reglamento de Arbitraje de la Cámara de Comercio Internacional ("CCI"). En él, si bien sólo se hace referencia al laudo cuando se alude a la etapa final del procedimiento, y se lo regula como el detonante de la terminación del proceso (de manera similar a lo que ocurre con la ley española de arbitraje, según lo ya explicado), queda algún espacio para interpretar que no todo laudo es final y definitivo, ya que el artículo 24 del reglamento se refiere al "laudo final" de los árbitros, como si hubiere uno o unos diferentes a éste.

\section{NACIONALIDAD DEL LAUDO}

Vistas estas nociones, queda claro entonces que, si bien la tendencia generalizada es la de entender por "laudo" la decisión final y definitiva de los árbitros, queda también algún espacio para pensar que, en un momento dado, y bajo ciertas normas o reglamentos internacionales, el calificativo de "laudo" podría hacerse extensivo también a otro tipo de pronunciamientos de los árbitros. En esa medida, lo que queda ahora es preguntarse cómo se define la "nacionalidad" de esos pronunciamientos que, bajo las perspectivas comentadas, puedan ser catalogados como "laudos", a fin de establecer, con base en ello, dónde se requie-

14. Artículo 4 de la Convención Interamericana sobre Arbitraje Comercial Internacional, suscrita en Panamá el 30 de enero de 1975. 
ren procedimientos como el del exequátur para hacer efectiva una determinada decisión y dónde no.

\subsection{Internacionalidad del Arbitraje}

Lo primero que hay que decir es que una cosa es el carácter de nacional, extranjero o internacional que pueda serle atribuido a un determinado procedimiento arbitral, y otra cosa bien distinta (aunque a veces suele confundírsele) es la nacionalidad del laudo. Empezando por el arbitraje mismo, una primera aproximación podría ser la de que, en efecto, como se trata de un "procedimiento", la calificación de nacional, extranjero o internacional que al arbitraje se le dé depende precisamente del origen del procedimiento al que las partes se hayan remitido para que los árbitros puedan tomar su decisión. En esta medida, un primer criterio para definir este aspecto podría consistir en que, si el procedimiento definido por las partes para la solución de un conflicto es el establecido por un reglamento internacional, el arbitraje será internacional. Hasta allí el asunto parece relativamente claro. La cuestión sin embargo comienza a complicarse cuando se mezclan otros elementos en el análisis. Por ejemplo, si las partes se remitieron a un reglamento típicamente internacional (como sería el de la CCI, por ejemplo), pero se trata de un conflicto entre dos partes domiciliadas en un mismo país y que no tiene ninguna relación con un lugar diferente, parecería absurdo calificar ese arbitraje como internacional.

La legislación colombiana, consciente de esta problemática, y tal como ha sido complementada con las interpretaciones de la Corte Constitucional, adoptó en la Ley 315 de 1996 un criterio que sigue muy de cerca los lineamientos establecidos en el artículo 1 de la Ley Modelo de UNCITRAL sobre Arbitraje Comercial Internacional, exigiendo la concurrencia de ciertos elementos para que el arbitraje pueda considerarse como verdaderamente internacional. Así, el artículo 1 de la Ley 315 dispone que será internacional el arbitraje cuando, además del pacto de 
las partes en ese sentido ${ }^{15}$, haya también un elemento de "internacionalidad", como sería el domicilio de las partes, el lugar de cumplimiento de las prestaciones sustanciales relacionadas con el litigio, el lugar del arbitraje ${ }^{16}$, la vinculación del asunto con los intereses de más de un estado o la afectación de intereses propios del comercio internacional ${ }^{17}$. Así las cosas, a la luz de la legislación colombiana es claro que la sola remisión a un reglamento internacional, o la sola escogencia de una sede distinta de Colombia como lugar del arbitraje (ver nota de pie de página número 16 anterior) no serían suficientes para determinar, por sí mismas, el carácter internacional de un arbitraje.

Ahora bien; yendo al fondo de nuestro asunto, hay que precisar lo siguiente: el hecho de que exista un arbitraje internacional no excluye de suyo la posible existencia de un laudo nacional. En otras palabras, resulta perfectamente posible que, a pesar de que el procedimiento arbitral reúna todas las condiciones para ser considerado como "internacional", según lo arriba descrito, pueda dicho procedimiento dar lugar a un laudo nacional. Para entender este punto a cabalidad, pasemos entonces a analizar el tema de la nacionalidad del laudo.

15. En relación con este punto, existe la discusión en cuanto a si las partes deben ser expresas en decir quc sc trata de un arbitraje internacional, o si por el contrario, ello puede deducirse del texto mismo de la cláusula, y, eventualmente, de las reglas a las cuales refieran el arbitraje. En nuestro criterio, esta última posición es la aceptable, por ser compatible con la flexibilidad propia del atbitraje internacional, y porque el querer de las partes, que desde luego es la piedra angular de toda cláusula arbitral, no solo es susceptible de reflejarse a través de manifestaciones expresas.

16. Sobre este punto, la Corte Constitucional Colombiana hizo importantes precisiones en la Sentencia C-347 de Julio 23 de 1.997 (MP: Jorge Arango Mejía). En este pronunciamiento, se dejó claro que no basta pactar que el arbitraje tendrá lugar en otro país para que haya "Arbitraje Internacional". Bajo la óptica jurisprudencial, si el elemento de "internacionalidad" va a ser el lugat del arbitraje, se requiere además que al menos una de las partes esté domiciliada en el extranjero (lo cual no implica que en. todo arbitraje internacional una de las partes tenga que ser extranjera o estar domiciliada en el extranjero; solamente si se va a hacer nso del criterio de localización del arbitraje como factor de intemacionalidad, habria entonces que complementarlo con otro elemento, como sería el domicilio en el exterior de una de las partes). De lo contrario, se abriria la puerta para "extraterritorializar", sin justificación ninguna, la solución de un conflicto netamente colombiano.

17. Este es un criterio eminentemente económico tomado de la jurispnudencia francesa, y que en términos generales, señala que los intereses del comercio internacional se afectan cuando hay movimiento transfronterizo de mercancias, bienes, capitales o servicios. 


\subsection{Nacionalidad del Laudo}

Dado que la definición en torno a la nacionalidad del laudo sólo es relevante en el marco de un conflicto que tenga elementos de "internacionalidad", según los hemos denominado a lo largo de este escrito, la determinación de dicha nacionalidad debe tener en cuenta lo que al efecto han establecido los instrumentos internacionales sobre la materia con aplicación en Colombia, y particularmente, la ya mencionada Convención de Nueva York, adoptada finalmente como parte de legislación colombiana mediante la Ley 39 de 1990.

De acuerdo con el criterio esbozado por el artículo 1 de la Convención de Nueva York, cuya aplicación, como ya se comentó en otro aparte de este escrito, se orienta al reconocimiento y ejecución de "sentencias arbitrales extranjeras", la nacionalidad del laudo está definida, en primer término, bajo una perspectiva eminentemente territorial. En efecto, el artículo citado menciona que la Convención se aplica a sentencias arbitrales dictadas en un territorio distinto al del estado donde se pide su reconocimiento, con lo cual la solución parece bastante simple: si el laudo fue proferido por fuera del territorio colombiano, se considera un laudo extranjero para su ejecución en Colombia, y por lo tanto, le serán aplicables las reglas y principios correspondientes; por el contrario, si el laudo fue proferido en Colombia, el laudo es, al menos en teoría, un laudo nacional para todos los efectos.

No obstante la aparente claridad de este principio, la Convención de Nueva York ha añadido otro criterio con el cual, ahí sí, surge alguna dificultad interpretativa. En efecto, además del criterio meramente territorial al que hemos hecho referencia, señala la Convención que su aplicación se extenderá "...también a las sentencias arbitrales que no sean consideradas como sentencias nacionales en el Estado en el que se pide su reconocimiento y ejecución...", ante lo cual cabe afirmar entonces que un laudo proferido en Colombia puede eventualmente, por alguna razón, "no ser 
considerado como sentencia nacional" en Colombia. Así por ejemplo, en el evento (improbable pero posible) en que dos extranjeros sin domicilio en Colombia celebren un contrato en el exterior para ser ejecutado por fuera del territorio colombiano, y acuerden deferir la solución de sus conflictos a un arbitramento bajo las reglas de la CCI en el territorio colombiano pero con aplicación de la ley de otro país, sería a todas luces evidente que el pronunciamiento que de ello resultare, a pesar de ser emitido en Colombia, no sería una sentencia nacional, puesto que ni las partes, ni su domicilio, ni el asunto objeto del litigio, ni las reglas procesales o sustanciales aplicables tendrían nada que ver con el territorio colombiano. En consecuencia, parecería claro que un pronunciamiento semejante debería tratarse en Colombia como un laudo extranjero, aplicándose para su reconocimiento y ejecución los principios de la Convención de Nueva York y los trámites propios del exequátur.

Teniendo en cuenta lo anterior, surge entonces aquí un nuevo criterio: un laudo será nacional o extranjero, no solo en razón del lugar donde sea proferido, sino también en razón de los puntos de contacto que pueda tener con una determinada jurisdicción. A partir de ello, fluye entonces otra conclusión: si bien no podemos llegar al extremo de decir, como se ha sostenido en algunos foros internacionales, que la escogencia del lugar del arbitraje es completamente irrelevante y estéril ${ }^{18}$, sí podemos

18. Ciertamente, el lugar del arbitraje no puede ser totalmente intrascendente, si se tiene en cuenta que, por ejemplo, de él depende la asistencia judicial que pueda requerirse para ciertas etapas del proceso. Para ilustrar el punto con mayor profundidad, vale la pena remitirse a dos artículos publicados por la Revista Internacional de Atbitraje: el primero, publicado en la edición No. 3 de la revista bajo el tituIo "La Escogencia del Lugar del Arbitraje", donde el autor Henri C. Álvarez formula importantes reflexiones sobre la materia, extendiendo la importancia del fugar del arbitraje hasta aspectos como la forma y contenido del laudo, la práctica de pruebas o el reconocimiento primario del laudo, para no ir muy lejos. El segundo, denominado "La Madurez del Arbitraje Comercial Internacional: de laudos extranjeros y laudos internacionales", en el que Dyalá Jiménez Figueres, con la autoridad que le confiere en estos temas su pasado desempento como funcionaria de la CCI, sostiene, entre otras cosas, que quien participa en ia definición de ta sede del arbitraje mediante una cláusula en la que ello se establezca, debe hacerlo responsablemente y sobre todo, con la plena conciencia de que ello tendrá ciertas implicaciones positivas y negativas: por una parte, servirá para pedit apoyo de los tribunales locales para adelantar el procedimiento arbitral, en caso de ser ello necesario, y por otra parte, servirá para establecer determinados controles sobre el laudo. ("Revista Internacional de Arbittaje" Universidad Sergio Arboleda, Comité Colombiano de Arbitraje y Legis; ediciones 4 y 5 , Bogotá, Julio - Diciembre de 2005 y Enero - Junio de 2006). 
al menos sostener que no es ella un factor determinante de la nacionalidad del laudo, y por ende, tampoco lo es para la fijación de todos los procedimientos aplicables a su reconocimiento y ejecución. Esta visión, acogida por los más modernos instrumentos de arbitraje internacional, constituye, a no dudarlo, una tendencia cada vez más fuerte en estas materias. Así por ejemplo, la Ley Modelo de UNCITRAL, a la que ya hemos hecho referencia, contempla en su artículo 35 que "...tn laudo arbitral, cualquiera que sea el país en que se haya dictado será reconocido como vinculante..." (el subrayado es nuestro), y en la misma línea, el Reglamento Suizo de Arbitraje Internacional, que es una de las más recientes elaboraciones al respecto ${ }^{19}$, consagra que a falta de acuerdo de las partes sobre la ley aplicable, los árbitros decidirán “...con arreglo a las normas juridicas que presenten la conexión más estrecha con el litigio..."20, más que con apego a las normas del lugar del arbitraje.

EI otro punto importante es el de los laudos "sin nacionalidad". A pesar de lo extravagante que a primera vista pueda aparecer la existencia de laudos sin vínculos de conexidad con un estado en particular, lo cierto es que en el escenario internacional sería perfectamente viable la existencia de un laudo a-nacional, dado que la nacionalidad no es un atributo propio, ni mucho menos necesario, de los laudos arbitrales. De ninguna manera resulta contrario a la complejidad propia de los negocios internacionales el hecho de que, eventualmente, existan partes con nacionalidades y domicilios diferentes, vinculadas por un negocio con desarrollos en diversos países, que para escoger un ámbito neutral para la solución de sus conflictos acuerden fijar como sede del arbitramento a otro país, bajo reglas definidas por un reglamento internacional como el de la CCI, la CIAC, la "American Arbitration Association", la "London Court of International Arbitration" o alguno similar. En una situación como ésta, lo que sí reñiría con toda lógica sería encasillar a ese laudo bajo un criterio de nacionalidad, puesto que precisamente es

19. El Reglamento data del año 2004.

20. Artículo 33 del Reglamento Suizo de Arbitraje Internacional. 
claro que lo que las partes quisieron fue desterrar de su relación este elemento, evitando así crear nexos entre su situación de conflicto y un estado en particular.

Ante un panorama como este, cabría preguntarse si los mecanismos diseñados por la Convención de Nueva York serían también aplicables a ese laudo sin nacionalidad. A primera vista, y si se toma el primero de los criterios de aplicación esbozados por la misma Convención, llegaríamos a la conclusión de que, si bien es viable que exista un laudo sin muchas conexiones con un estado determinado, no sería posible la existencia de un laudo sin que al menos, su lugar de expedición sea medianamente claro $^{21}$. Bajo esta concepción entonces, que es la que hemos denominado atrás como "criterio de territorialidad", el laudo siempre tendría una nacionalidad, cual sería la del lugar de su expedición, y en consecuencia, la Convención de Nueva York sería aplicable siempre que el laudo se pretenda hacer valer en un país distinto de aquel donde fue proferido. No obstante lo anterior, y como ya lo analizamos, existe otro criterio de aplicación fijado en la misma Convención, cual es el de tratar como una "sentencia arbitral extranjera" (y por tanto, objeto de la Convención) a aquella que no sea considerada por un estado como una sentencia nacional. En un ejemplo como el propuesto entonces, sería probable que ningún estado considerara como suyo el laudo en cuestión, y en consecuencia, a pesar de ser un laudo a-nacional, sería también un laudo "extranjero" (como lo exige la Convención para efectos de su aplicación) frente a todos los países donde pretendiera dársele aplicación.

21. En cualquier caso, en arbitraje internacional también resulta posible ( $y$ ocurre con frecuencia) que los árbitros no estén fisicamente presentes en un mismo lugar al proferirse el laudo. 


\section{ReFLeXIONES Finales}

Si bien no existen nociones establecidas, claras y uniformes sobre qué es un laudo o cómo se define su nacionalidad para efectos de definir las normas que pueden serle aplicables, especialmente en lo referente a su reconocimiento y ejecución, sí hay una tendencia más o menos generalizada a tratar como laudo únicamente a las decisiones de los árbitros que resuelven el fondo del asunto, y a contemplar la nacionalidad de una decisión arbitral más desde la perspectiva de sus relaciones de conexidad con los distintos estados, que con el sitio donde haya tenido lugar el procedimiento arbitral o donde se haya proferido la decisión de que se trate.

Más allá de lo anterior sin embargo, lo importante es entender que la ausencia de criterios rígidos y de definiciones establecidas en estas materias, antes que constituirse en un factor de confusión, es un elemento más de la flexibilidad propia de la justicia arbitral. Tratar de encasillar dentro de los límites de una definición legal (especialmente si proviene de los ordenamientos nacionales) lo que puede considerarse o no como laudo, o los criterios determinantes para la fijación de su nacionalidad, le haría un daño enorme a la institución arbitral, pero sobre todo, impediría de suyo que los conflictos que por su naturaleza misma tengan un carácter transnacional trasciendan los límites de los estados nacionales.

BOGOTÁ, JUNIO DE 2009

$\left.{ }^{*}\right)$ Socio de Prieto \& Carrizosa - Colombia 